Eurographics Symposium on Rendering (2006)

Tomas Akenine-Möller and Wolfgang Heidrich (Editors)

\title{
Visual Chatter in the Real World
}

\author{
Shree K. Nayar and Gurunandan G. Krishnan \\ Department of Computer Science \\ Columbia University, New York, U.S.A.
}

\begin{abstract}
When a scene is lit by a source of light, the radiance of each point in the scene can be viewed as having two components, namely, direct and global. Recently, an efficient separation method has been proposed that uses high frequency illumination patterns to measure the direct and global components of a scene. The global component could arise from not only interreflections but also subsurface scattering within translucent surfaces and volumetric scattering by participating media. In this paper, we use this method to measure the direct and global components of a variety of natural and man-made materials. The computed direct and global images provide interesting insights into the scattering properties of common real-world materials. We have also measured the two components for a $3 D$ texture as a function of lighting direction. This experiment shows that the global component of a BTF tends vary smoothly with respect to the lighting direction compared to the direct component of the BTF. Finally, we apply the separation method to a translucent object for different imaging and illumination scales (resolutions). The results obtained show how the BSSDRF of the object gradually reduces to a BRDF as one goes from fine to coarse scale. All the measurement results reported here, as well as several others, can be viewed as high resolution images at http://www1.cs.columbia.edu/CAVE/projects/separation/separation.php.
\end{abstract}

Categories and Subject Descriptors (according to ACM CCS): I.3.7 [Computer Graphics]: Three-Dimensional Graphics and Realism I.4.1 [Image Processing and Computer Vision]: Digitization and Image capture

\section{Introduction}

When a scene is lit by a source of light, the radiance of each point in the scene can be viewed as having two components, namely, direct and global. The direct component arises from the direct illumination of the scene point by the light source. The global component results from the illumination of the point due to scattering of light from other points in the scene. The global illumination can result from a variety of effects, including, interreflections, subsurface scattering and volumetric scattering. Recently, the authors, in collaboration with Michael Grossberg at the City University of New York and Ramesh Raskar at MERL, have proposed efficient methods that use high frequency illumination patterns to separate the direct and global components of a scene lit by a single light source [NKGR06]. The illumination patterns include shifted checkerboard patterns, shifted sine patterns, shadows cast by a translating line occluder and shadows cast by a rotating mesh occluder.

The goal of this paper is to present separation results for a variety of real-world materials and objects. For this, we have used a 1280x720 DLP projector and a 1024x728 color camera. We have used the shifted checkerboard method [NKGR06] with checker sizes in the range of $4 \times 4$ to $8 \times 8$ (in projector pixels). In the remaining of this paper, we will show our experimental results. In each figure, the details of the result are discussed in the caption. We conclude with separation experiments in which the source direction, the resolution of the camera and the resolution of the illumination pattern are varied. These experiments show the decomposition of a BTF [DvGNK99] into its direct and global components, and the transition of a BSSRDF [JMLH01] to a BRDF [Nic70] as the scale of observation and illumination varies from fine to coarse.

The examples shown here illustrate how real-world objects interact with light as well as optically interact with each other (hence the term "visual chatter"). Some of these interactions confirm our intuitions on how global illumination works. Others reveal surprising effects and provide new insights into the scattering properties of commonplace materials.

(C) The Eurographics Association 2006. 
Scene

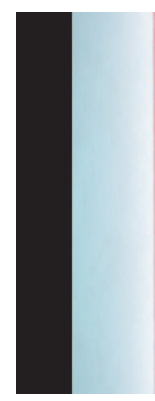

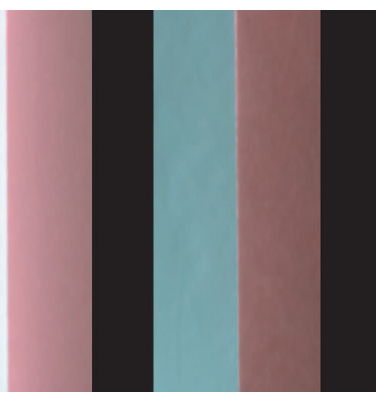
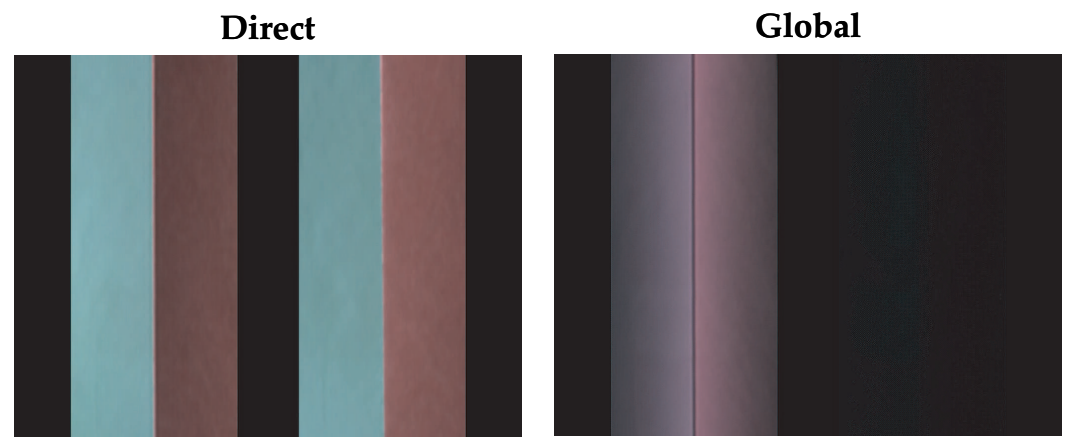

Figure 1: Concave and Convex V-Grooves: This scene includes two identical V-grooves, except that the left one is concave and the right one is convex. The two sides of each groove are made of matte paper (white on left side and pink on the right). As expected, the convex groove has no global illumination while the concave one has a strong global component due to diffuse interreflections that increase toward the edge of the groove. The interreflections cause the color of one side to "bleed" into the other. Several studies have been conducted in psychophysics that show that interreflections play an important role in the perception of shape and color [Gil79] [BKH99].
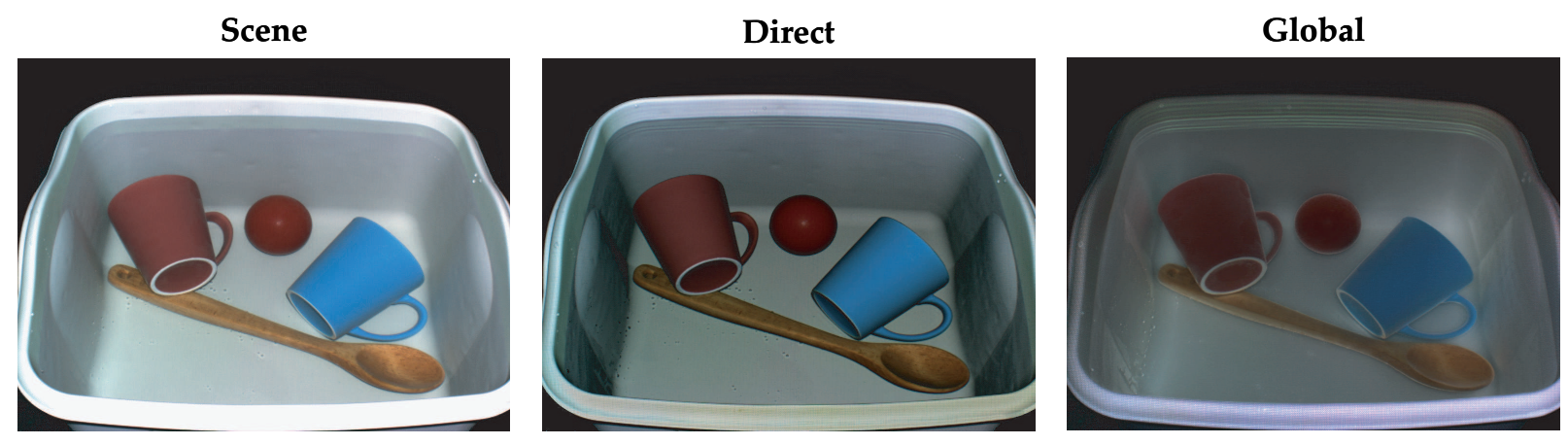

Figure 2: Kitchen Sink: This scene includes objects in a sink filled with water. Since the water is clear it serves as a fully transparent medium and does not influence the scattering effects. It is worth noting that the computed direct image looks like a synthetic image rendered using a single-bounce rendering package such as OpenGL. All the interreflections between the sink and the objects are observed in the global image. Notice the strong interreflections at the edges and corners of the sink and the occluding boundaries of the curved objects.
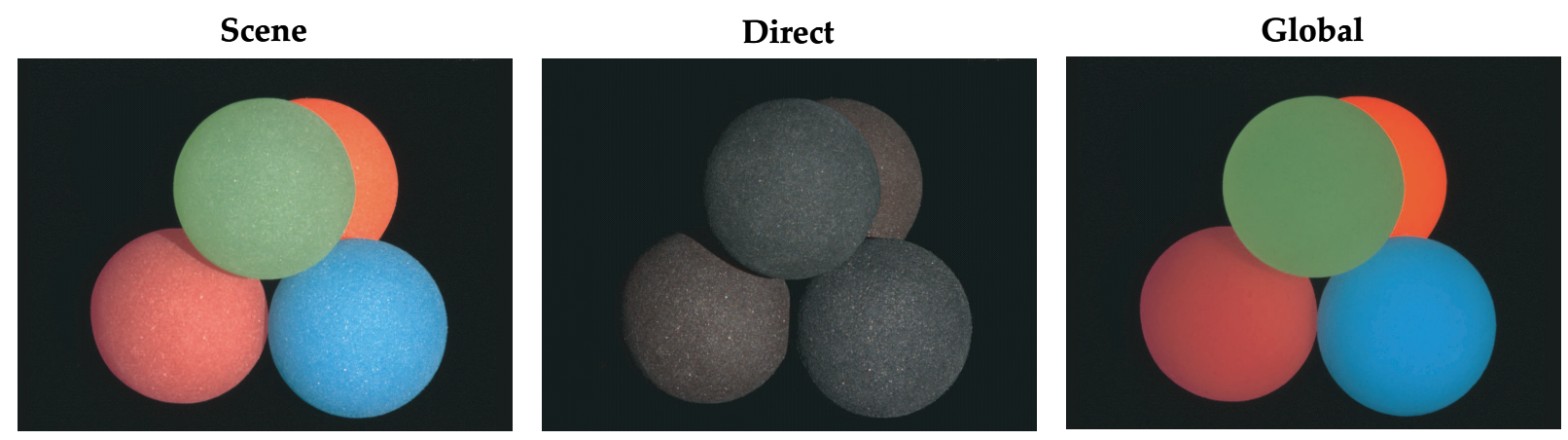

Figure 3: Translucent Colored Balls: The balls in this scene exhibit very strong subsurface scattering which causes them to "glow" under virtually any illumination [JMLH01] [GLL*04]. We see that all the subsurface scattering is captured in the global image. Perhaps, due to strong multiple scattering, the global images of the balls have very little shading, causing the balls to appear like flat discs (particularly, the green ball). On the other hand, the direct component reveals the spherical shapes of the balls and the roughness of their surfaces. 

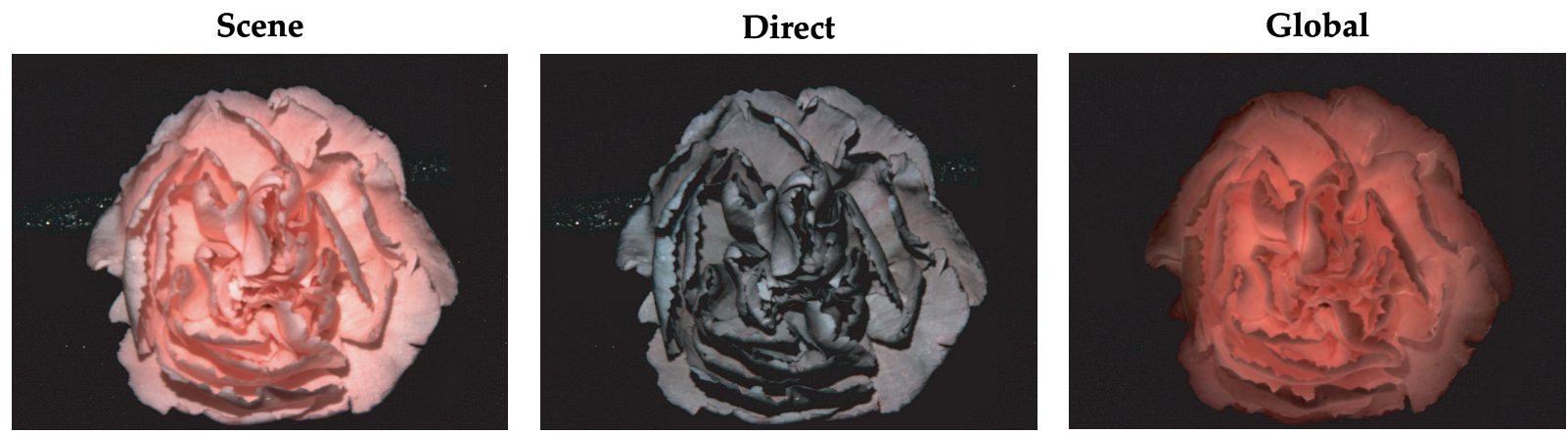

Figure 4: Pink Carnation: In the case of this flower, we see that in the direct image the shadows cast by the petals on each other are strong and the petals themselves appear grayish and somewhat listless. As a result, the direct image looks more like that of a synthetic flower than a natural one. It is interesting to note that most of the color of the flower arises from global effects. These include the interreflection of light between the petals as well as the diffusion of light through the petals. Both these effects cause a "sharpening" of the spectral distribution of the light [FDH91]. As a result, in this example, the color of the light gets more reddish after each bounce or diffusion.

Scene

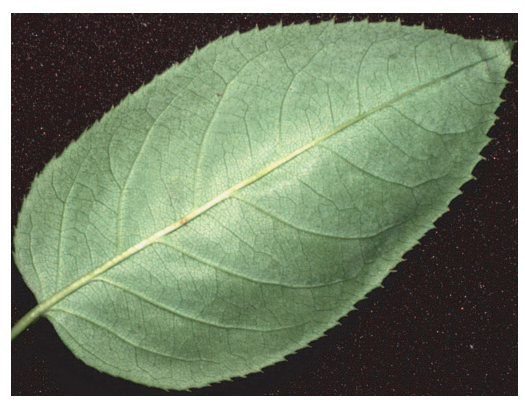

Direct

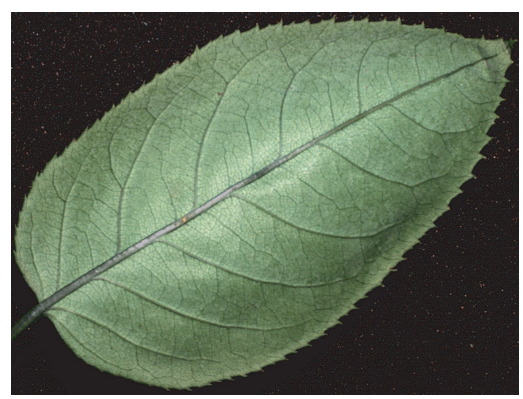

Global

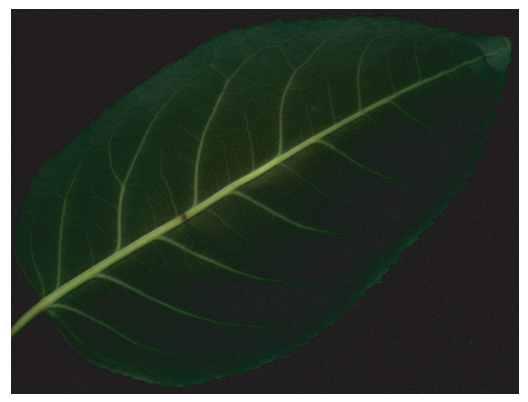

Figure 5: Hybrid Tea Rose Leaf: Although the leaf is thin, it exhibits a noticeable global component over its entire area. This is because the entire leaf has a spongy mesophyll layer, beneath the upper epidermis and palisade mesophyll layers, that exhibits subsurface scattering. In particular, the global component is strong for the veins of the leaf. This is probably because the veins are translucent as they are made of vascular tissues that carry water, minerals and sap. See [PSOH03] for details on the anatomy of the leaf.
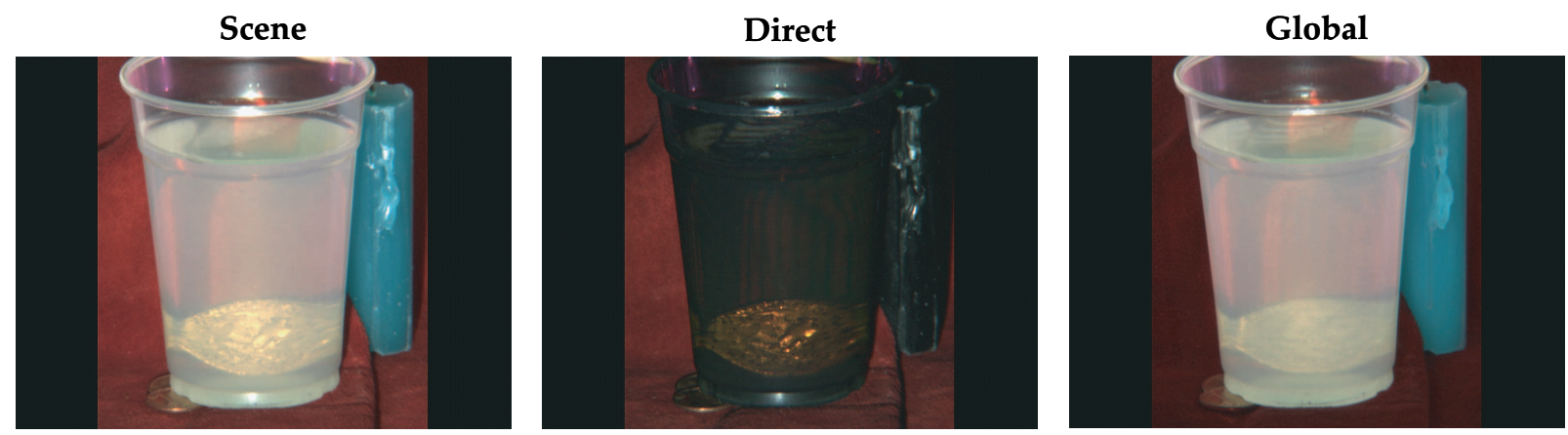

Figure 6: Plastic Cup with Milky Water and Coin: In this example, the global image includes the volumetric scattering [Cha50] [Ish78] of light by the milky water (referred to as "airlight" in atmospheric optics [Mid52] [McC76]) in the cup as well as the secondary illumination of the copper coin by the milky water [SRNN05]. The direct component includes the specular highlights on the copper coin due to direct illumination of the coin by the source. This component is attenuated (referred to as "direct transmission" in atmospheric optics [Mid52] [McC76]) by the milky water as it makes its way to the camera. The wax candle on the right has a strong subsurface scattering component. As a result, all of its color is captured in the global image and the direct image only includes the surface reflection (highlights) from the candle.

(c) The Eurographics Association 2006. 
Scene

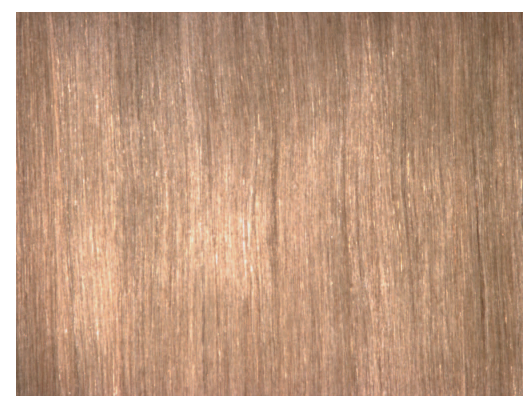

Direct

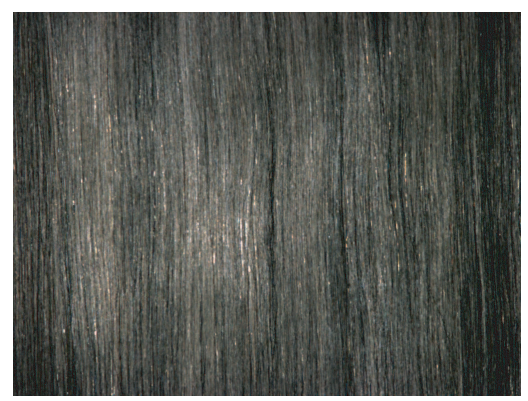

Global

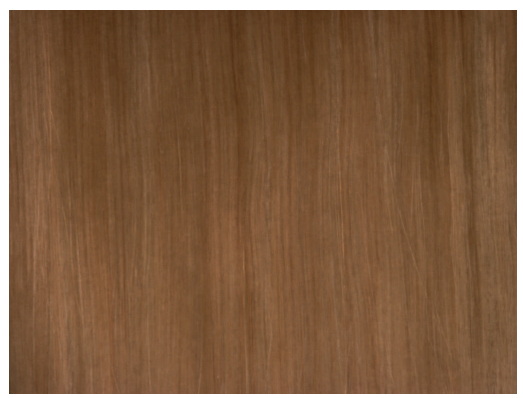

Figure 7: Blonde Hair: In this example, we see how blonde hair decomposes into its direct and global components. It is interesting to note that the direct component makes the blonde hair look like dark tan hair. Furthermore, the direct component reveals the complex BRDF of individual hair fibers, which is due to the nested-cone structure of each fiber [SGF77] [BS91] [LKK00] [MJC*03]. One can observe the complex structures of the specular highlights as well as rainbow scattering effects that are known to occur in hair fibers. In contrast, the global component has the appearance of a flat texture such as that of finished wood [MWAM05]. The global component arises from multiple reflections of light between the hair fibers [MJC*03] and is seen to account for the color of the hair.
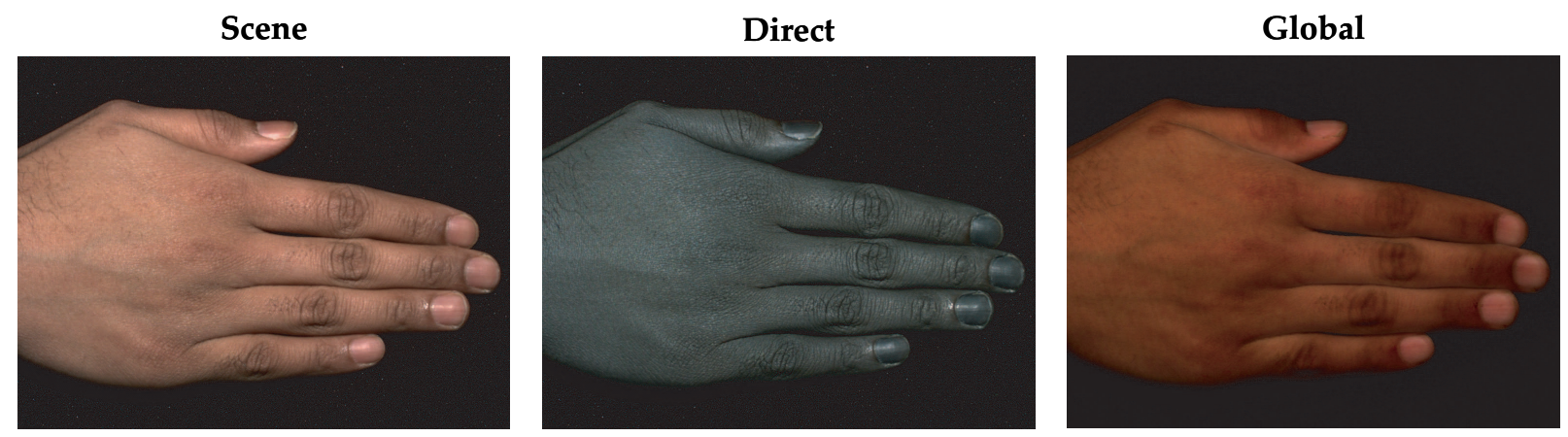

Figure 8: Hand: Here, we show separation results for the hand of an Asian Indian male. Notice how the direct component mainly includes the surface reflection due to oils and lipids on the skin. It also reveals the details of the micro-geometry of the skin surface [UKM*96] [HEG01] [KP03] [CDMR04]. Most of the color of the skin comes from subsurface scattering, as seen in the global image [HK93] [DHT*00] [JMLH01] [TOS*03] [WMP* 05]. In contrast to the direct component, the global component does not reveal the roughness of the skin's surface and only includes albedo variations. The details of the geometry and optics of skin can be found in [INNO5].

Scene

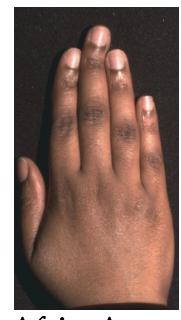

Afric. Amer

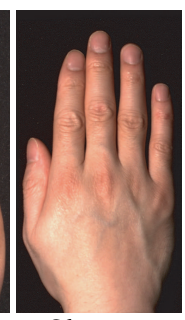

Chinese

Male

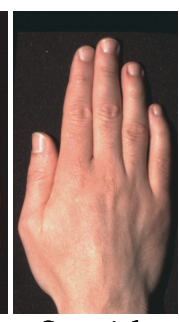

Spanish

Male
Direct

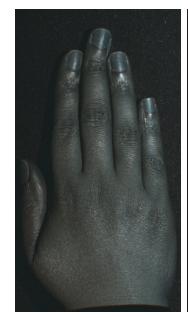

Afric. Amer

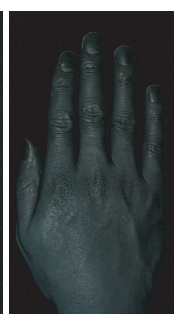

Female

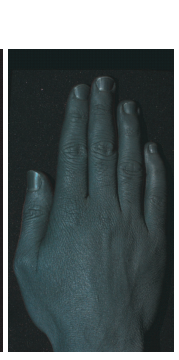

Spanish

Male

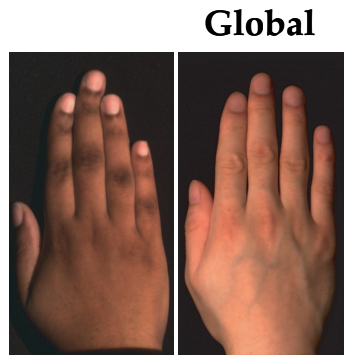

Afric. Amer. Chinese Female

Male

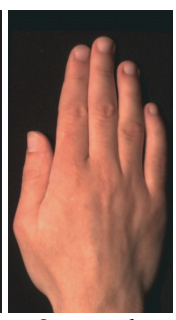

Spanish

Male

Figure 9: Hands of Different Races: The separation results for three more hands of people of different nationalities: African America female (left), Chinese male (middle) and Spanish male (right). In these specific hands, the micro-geometry of the skin is quite different, as seen from the direct component. Once again, the colors of the skin come from the global component and depend on the melanin and hemoglobin levels within the skin [TOS*03] [INN05]. 

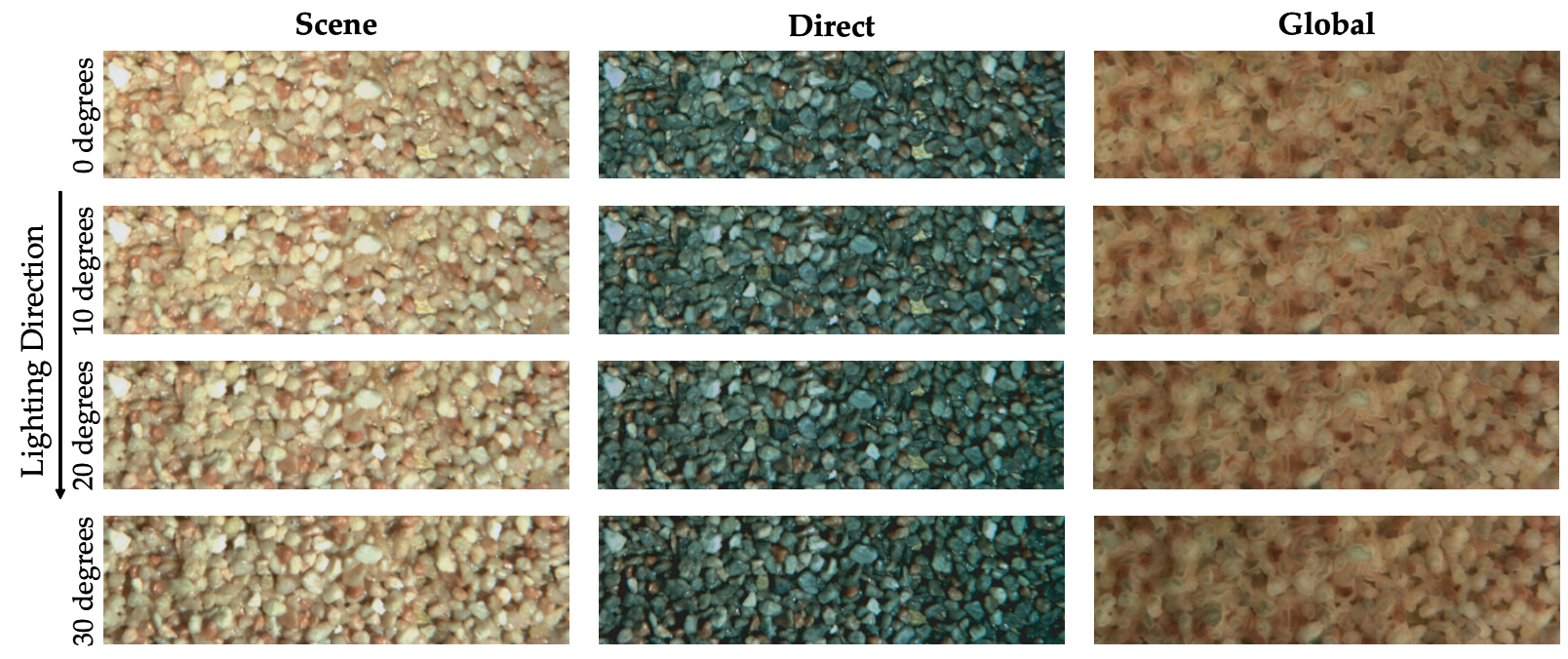

Figure 10: Direct and Global BTF of Pebbles: For this sample of pebbles taken from the CURET database [DvGNK99] we varied the source direction in a plane between -30 and 30 degrees in steps of 10 degrees (where 0 degrees corresponds to the viewing direction), and performed the separation for each source direction. The end result is a decomposition of the BTF [DvGNK99] into its direct and global components. Notice how the direct images look like real images of dark pebbles. The global images capture the subsurface scattering within individual pebbles as well as the complex interreflections between neighboring pebbles. As the source direction varies, the direct images vary significantly within local regions as the shadows shift and the shading changes. However, the global image varies more smoothly with respect to the lighting direction. This confirms our intuition that the variation in global illumination tends to be smooth with respect to lighting direction.
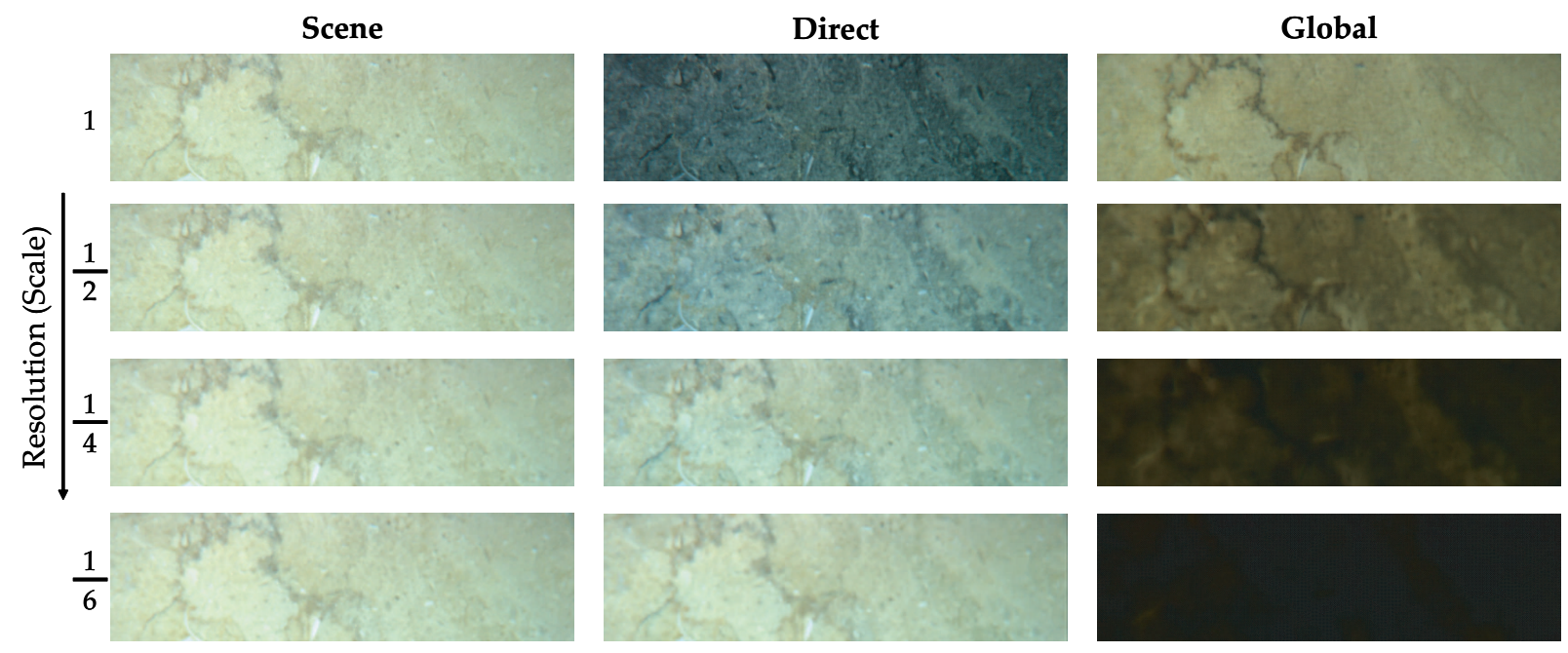

Figure 11: BSSRDF and BRDF of Marble as a Function of Scale: In this example, we show separation results for a marble tile for different resolutions of imaging and illumination. For scale $=1$, the resolution of the camera was set at the native resolution of $1024 x 768$ and the separation was done with a checkerboard pattern with checkers of $4 x 4$ projector pixels. For lower scales of $1 / n$, the resolution of the camera was reduced by aggregating nxn pixels and the checker size of the illumination pattern was increased to $4 n \times 4 n$. At scale $=1$, we see a slight direct component and a dominant global component [JMLH01] [GLL*04]. In contrast, at scale $=1 / 6$, we see that the global component is nearly zero everywhere and the direct component is almost equal to the original image. This example shows how the BSSRDF of a translucent object dominates at fine scales, but reduces to a $B R D F$ as one goes to coarser scales. 


\section{Acknowledgments}

This research was supported by a grant from the Office of Naval Research (No. N000140510188).

\section{References}

[BKH99] Bloj M. G., Kersten D., Hurlbert A. C.: Perception of Three-Dimensional Shape Influences Colour Perception through Mutual Illumination. Nature 402 (1999), 877-879. 2

[BS91] Bustard H., Smith R.: Investigation into the Scattering of Light by Human Hair. Applied Optics 24, 30 (1991), 3485-3491. 4

[CDMR04] Cula O. G., Dana K. J., Murphy F. P., RAO B. K.: Bidirectional Imaging and Modeling of Skin Texture. IEEE Trans. on Biomedical Eng. 51, 12 (Dec 2004), 2148-2159. 4

[Cha50] ChandrasekhaR S.: Radiative Transfer. Clarendon Press, Oxford, UK, 1950. reprinted by Dover Publications, 1960. 3

[DHT*0] Debevec P., Hawkins T., Tchou C., Duiker H., SAROKIN W., SAgar M.: Acquiring the Reflectance Field of a Human Face. In Proc. of $S I G$ GRAPH (July 2000), ACM Press. 4

[DvGNK99] DANA K. J., VAN GINNEKEN B., NAYAR S. K., KoEnderinK J. J.: Reflectance and Texture of Real World Surfaces. ACM Trans. Graph. 18, 1 (Jan. 1999), 1-34. 1, 5

[FDH91] Funt B. V., Drew M. S., Ho J.: Color Constancy from Mutual Reflection. Int. Jour. of Comp. Vision 6, 1 (1991), 5-24. 3

[Gil79] Gilchrist A.: The Perception of Surface Blacks and Whites. Scientific American 240 (1879), 112-123. 2

[GLL*04] Goesele M., Lensch H., LANG J., Fuchs C., SEIDEL H.: DISCO: Acquisition of Translucent Objects. In Proc. of SIGGRAPH (2004), ACM Press, pp. 835-844. 2, 5

[HEG01] Haro A., Essa I. A., Guenter B. K.: Realtime, Photo-realistic, Physically Based Rendering of Fine Scale Human Skin Structure. In Proc. of 12th Eurograph. Work. on Rend. (2001), Springer-Verlag, pp. 53-62. 4

[HK93] Hanrahan P., Krueger W.: Reflection from Layered Surfaces due to Subsurface Scattering. In Proc. of SIGGRAPH (1993), ACM Press, pp. 165-174. 4

[INN05] Igarashi T., Nishino K., Nayar S. K.: The Appearance of Human Skin. Tech. Report, Dept. of Comp. Science, Columbia Univ. CUCS-024-05 (Jun 2005). 4

[Ish78] ISHIMARU A.: Wave Propagation and Scattering in Random Media. Academic Press, 1978. 3

[JMLH01] Jensen H. W., Marschner S. R., Levoy M., Hanrahan P.: A Practical Model for Subsurface Light Transport. In Proc. of SIGGRAPH (2001), ACM Press, pp. 511-518. 1, 2, 4, 5
[KP03] Koenderink J. J., Pont S.: The Secret of Velvety Skin. Mach. Vision Appl. 14, 4 (2003), 260-268. 4

[LKK00] LU R., Koenderink J. J., Kappers A. M. L.: Specularities on Surfaces with Tangential Hairs or Grooves. Comp. Vision and Img. Understanding 78 (2000), 320-335. 4

[McC76] MCCARTney E. J.: Optics of the Atmosphere: Scattering by Molecules and Particles. John Wiley and Sons, New York, 1976. 3

[Mid52] Middleton W. E. K.: Vision through the Atmosphere. Univ. of Toronto Press, 1952. 3

[MJC*3] Marschner S. R., Jensen H. W., CAMMARANo M., Worley S., HANRAhan P.: Light Scattering from Human Hair Fibers. Proc. of SIGGRAPH 22, 3 (2003), 780-791. 4

[MWAM05] MARSChNer S. R., Westin S. H., ARBree A., Moon J. T.: Measuring and Modeling the Appearance of Finished Wood. Proc. of SIGGRAPH 24, 3 (2005), 727-734. 4

[Nic70] Nicodemus F. E.: Reflectance Nomenclature and Directional Reflectance and Emissivity. Applied Optics 9 (1970), 1474-1475. 1

[NKGR06] Nayar S. K., Krishnan G., GrossberG M. D., RASKAR R.: Fast Separation of Direct and Global Components of a Scene using High Frequency Illumination. Proc. of SIGGRAPH (July 2006), 145-156. 1

[PSOH03] PurVes W. K., SAdaVA D., Orians G. H., Heller H. C.: Life: The Science of Biology, Seventh Edition. Sinauer Associates and W. H. Freeman, 2003. 3

[SGF77] Stamm R. F., Garcia M. L., Fuchs J. J.: The Optical Properties of Human Hair I. Fundamental Considerations and Goniophotometer Curves. J. Soc. Cosmet. Chem. 28 (1977), 571-600. 4

[SRNN05] SUN B., RAMAMOORTHI R., NARASIMHAN S., NAYAR S.: A Practical Analytic Single Scattering Model for Real Time Rendering. Proc. of SIGGRAPH 24, 3 (Jul 2005), 1040-1049. 3

[TOS*03] Tsumura N., OJima N., Sato K., ShiRaishi M., Shimizu H., Nabeshima H., AKazaki S., HORI K., MIYAKE Y.: Image-based Skin Color and Texture Analysis/Synthesis by Extracting Hemoglobin and Melanin Information in the Skin. In Proc. of SIGGRAPH (2003), ACM Press, pp. 770-779. 4

[UKM*96] UChida T., Komeda T., Miyagi M., Koyama H., Funakubo H.: Quantification of Skin Aging by Three-Dimensional Measurement of Skin Surface Contour. In Systems, Man, and Cybernetics., IEEE Int. Conf. (1996), vol. 1, pp. 450-455. 4

[WMP*05] Weyrich T., Matusik W., Pfister H., Lee J., NGan A., Jensen H. W., Gross M.: A Measurement-Based Skin Reflectance Model for Face Rendering and Editing. None TR2005-071 (Jul 2005). 4 